\title{
Can contemporary trials of chemotherapy for HER2-negative metastatic breast cancer detect overall survival benefit?
}

This article was published in the following Dove Press journal:

Cancer Management and Research

\author{
Sherko Kümmel' \\ Christian Jackisch ${ }^{2}$ \\ Volkmar Müller ${ }^{3}$ \\ Andreas Schneeweiss ${ }^{4}$ \\ Sandra Klawitter ${ }^{5}$ \\ Michael P Lux ${ }^{6}$ \\ 'Breast Unit Essen, Kliniken Essen- \\ Mitte, Essen, Germany; ${ }^{2}$ Department \\ of Obstetrics and Gynecology, Sana \\ Klinikum Offenbach, Offenbach, \\ Germany; ${ }^{3}$ Department of Gynecology, \\ University Medical Center Hamburg- \\ Eppendorf, Hamburg, Germany; \\ ${ }^{4}$ Division of Gynecologic Oncology, \\ National Center for Tumor Diseases, \\ University Hospital, Heidelberg, \\ Germany; ${ }^{5}$ Medical Affairs - \\ Biostatistics and Epidemiology, Roche \\ Pharma AG, Grenzach-Wyhlen, \\ Germany; ${ }^{6}$ Department of Gynecology \\ and Obstetrics, Erlangen University \\ Hospital, Comprehensive Cancer \\ Center Erlangen-EMN, Friedrich- \\ Alexander University Erlangen- \\ Nuremberg, Erlangen, Germany
}

Correspondence: Sherko Kümmel Breast Unit Essen, Kliniken Essen-Mitte, Henricistrasse 92, 45I33 Essen, Germany $\mathrm{Tel} / \mathrm{fax}+4920117433001$

Email S.Kuemmel@kliniken-essen-mitte.de
Purpose : Although several trials have demonstrated improved progression-free survival (PFS) with first-line regimens for HER2-negative metastatic breast cancer $(\mathrm{mBC})$, overall survival (OS) benefit is elusive. We calculated required sample sizes to power for OS using published data from recent $\mathrm{mBC}$ trials.

Patients and methods : Randomized superiority trials of first-line chemotherapy/targeted therapy for HER2-negative $\mathrm{mBC}$ including $>150$ patients, meeting the primary efficacy objective, and published in 2000-2018 were identified. The sample sizes required to power for PFS and OS were calculated retrospectively for each trial using observed results and study/recruitment follow-up durations ( $\alpha=0.05$, two-sided log-rank test, $80 \%$ power), and summarized as a factor (x) relative to actual sample size.

Results : Nine of 13 identified trials reported all information required for retrospective sample size calculation. Six had sample sizes larger than required to demonstrate a significant PFS benefit but all would have required larger sample sizes to demonstrate significant OS benefit with the observed results. In ten trials, the required sample size was $\geq 5$-fold larger to power for OS than PFS.

Conclusion: Designing trials to test potential new treatments for HER2-negative mBC is challenging, requiring a balance of regulatory acceptability, feasibility, and realistic medical assumptions to calculate sample sizes. Powering for OS is particularly difficult in heterogeneous populations with long postprogression survival, potential crossover, heterogeneous poststudy therapy, and evolving treatment standards. Validated surrogate endpoints are critical. Ongoing trials of cancer immunotherapy (new mode of action) in triple-negative mBC (more homogeneous, shorter OS and postprogression survival, fewer treatment options) may show a new pattern.

Keywords: progression-free survival, overall survival, endpoint, metastatic breast cancer, clinical trial, regulatory authorities

\section{Introduction}

In metastatic breast cancer ( $\mathrm{mBC})$, selection of the most appropriate endpoint for clinical trials is becoming increasingly important when evaluating new first-line therapies. In HER2-positive mBC, for which a number of targeted agents exist, several trials across treatment settings have demonstrated overall survival (OS) benefits from HER2directed therapies. ${ }^{1}$ In HER2-negative $\mathrm{mBC}$, however, where the target is less clear and patient selection is more challenging, progression-free survival (PFS) benefits have rarely translated into statistically significant OS benefits. To date, no Phase III trial evaluating antiangiogenic agents, cyclin-dependent kinase 4/6 inhibitors, mTOR inhibitors, or poly(adenosine diphosphate-ribose) polymerase inhibitors has shown a 
statistically significant OS improvement. OS is considered an unambiguous endpoint and is the global gold standard for demonstrating clinical benefit. However, extending life is not necessarily valuable if accompanied by significant quality of life (QoL) deterioration. Other disadvantages of OS as a primary endpoint are bias caused by treatment evolution during long studies, the diluting effect of crossover, numerous heterogeneous subsequent treatment lines, and the need for large patient numbers and/or long follow-up before obtaining results. This is particularly problematic in first-line trials, in which patients typically receive multiple treatment lines after progression. ${ }^{2,3}$ Consequently, authorities including the European Medicines Agency accept PFS as a relevant endpoint and approve drugs based on PFS benefit.

The correlation between PFS and OS appears to be less robust in settings with longer postprogression survival and/or effective subsequent therapies, ${ }^{4-6}$ whereas in later treatment lines, the likelihood of showing an OS benefit increases. ${ }^{7,8}$ However, a recent analysis of 40 randomized controlled trials in HER2-negative hormone receptor-positive $\mathrm{mBC}$ indicated a significant association between PFS/time to progression (TTP) and OS, irrespective of treatment line. ${ }^{9}$ To explore this topic further, we used published data from contemporary HER2-negative $\mathrm{mBC}$ trials to calculate the sample sizes required to power for OS compared with sample sizes actually used. Based on our findings, we discuss the challenges of designing trials in HER2-negative $\mathrm{mBC}$, where powering for OS is sometimes unrealistic, unfeasible, or unfundable, with the aim of improving future trial planning and design.

\section{Design}

Clinical trials were identified from a systematic search of MEDLINE (details in Table S1) using the following criteria: randomized superiority trials; first-line chemotherapy or targeted therapy for HER2-negative mBC; >150 patients; meeting the primary efficacy objective ("positive" trials); and published in English in a peer-reviewed journal between January 1, 2000 and February 15, 2018.

The sample sizes required to power for PFS/TTP and OS were calculated retrospectively for each trial using the observed median PFS/TTP and median OS in the treatment groups for treatment effect, the actual recruitment period, and the actual total study duration $(\alpha=0.05$, two-sided logrank test, $80 \%$ power). Dropout rates were not considered for sample size calculation. nQuery Advisor (version 7.0; Statistical Solutions Ltd, Cork, Ireland) was used for sample size calculations. If information on the total study duration was missing, we chose a simple pragmatic assumption that the study period was one-third longer than the recruitment duration.

The retrospectively calculated sample sizes were summarized as a factor ( $\mathrm{x}$ ) relative to the actual sample size. $\mathrm{x}<1$ would require $\mathrm{x}$-fold fewer cases to show a significant benefit, whereas $x>1$ required $x$-fold more cases.

\section{Results \\ Analysis data set}

Thirteen trials met the selection criteria (Table 1). Of these, nine reported all information required for retrospective sample size calculation. In four reports (all published before 2006), insufficiently described study duration made it difficult or impossible to understand fully the statistical assumptions for sample size calculation. Only one trial had OS as the primary endpoint.

In most trials, the HRs showed a stronger treatment effect on PFS/TTP than OS (Figure 1). Four trials showed statistically significantly improved OS. ${ }^{12,14,15,24}$

\section{Retrospective sample size calculation}

Table 2 shows the retrospectively calculated sample sizes required to show PFS/TTP and OS benefit with the observed data compared with the actual sample sizes. According to these calculations, six of 13 trials had sample sizes larger than required to demonstrate a significant PFS benefit. However, all would have required a larger sample size to demonstrate a significant OS benefit with the observed results. The increase in sample size ranged from 1.2-fold to 2,460-fold. In nine of the 12 trials with OS information, the calculated required sample size to demonstrate a significant OS benefit with the observed OS results was at least fivefold greater than the actual sample size. Figure 2 summarizes the sample size increase required to show a significant OS benefit with the reported data.

In all but one trial, a larger sample size would be required to show OS than PFS benefit. In 10 of the 12 trials with available OS results, the sample size required to power for OS was at least fivefold larger than that needed to power for PFS.

\section{Discussion}

Our analyses suggest that in the first-line HER2-negative $\mathrm{mBC}$ setting, it is a high hurdle to conduct a trial with adequate power to detect an OS improvement. Sample sizes to power for OS are usually extremely large and substantially larger than required to power for PFS.

The generally larger PFS than OS treatment effect in HER2-negative $\mathrm{mBC}$ is consistent with a recently reported 
study across various tumor types. ${ }^{27}$ Our findings are also consistent with reports in the literature suggesting that demonstrating an OS benefit is becoming increasingly unrealistic in contemporary clinical trials. ${ }^{2} \mathrm{~A}$ trial without crossover may answer the question of OS most cleanly. However, if the investigational agent has shown clear activity, the possibility of crossover has to be discussed. An Independent Data Monitoring Committee may feel obliged to stop a trial because of a clear signal, but it will then be impossible to conclude on the secondary endpoint of OS. Furthermore, a second trial of the same agent cannot be conducted after proven benefit because it is difficult to consent patients to be randomized between an experimental agent and a control arm known to be inferior. At times of rapid innovation, endpoints allowing prompt application of therapy optimization to standard clinical care are required. Therefore, it is important to determine whether progression-based endpoints are suitable for demonstrating utility. Available endpoints include PFS, TTP, and time to treatment failure. These allow earlier provision of study results and can be more sensitive indicators of treatment benefit because they are not affected by further treatment lines or crossover. ${ }^{28,29}$ Another benefit is comparability, as PFS is currently the most commonly used primary endpoint in Phase III trials. However, there is no clear evidence that PFS is a surrogate for OS.

In a recent analysis of PFS and OS in 58 randomized Phase II/III trials evaluating first-line systemic therapy for HER2-negative hormone receptor-positive $\mathrm{mBC}$, several factors besides first-line therapy were reported to influence OS. ${ }^{30}$ These included prior endocrine therapy, prior (neo)adjuvant chemotherapy, types and lines of postprogression therapy, as well as disease characteristics associated with prognosis. Geographic region also influenced OS, presumably because of differences in healthcare patterns, management, and access in different countries.

In our analysis, the trial in which the actual sample size and the retrospectively calculated sample size for OS were most similar was IMELDA, a maintenance trial evaluating the addition of capecitabine to maintenance bevacizumab after bevacizumab/taxane induction therapy as a new treatment approach. ${ }^{24}$ In these patients already demonstrating chemosensitivity to induction therapy, switching to capecitabine before progression potentially anticipates development of resistance. In IMELDA, a significant OS benefit was demonstrated with a relatively small sample size but the retrospectively calculated sample size suggested that a larger sample size was needed. This is explained by differences in the methodology used for sample size calculation compared with the trial analysis method. Importantly, sample size calculation is only an estimation. Conversely, trial outcome is not proof and there is $5 \%$ error for demonstrating a significant benefit.

There appeared to be a gradual increase in median OS in the investigational arm over time (Table 2). Such cross-trial comparisons have obvious limitations, particularly when including maintenance vs treatment strategies. Nevertheless, median OS with experimental therapy remained $<2$ years in all trials evaluating chemotherapy alone, crossing the 2-year threshold only with the introduction of targeted therapy (bevacizumab). This presumably reflects not only treatment effect but also earlier diagnosis, better disease management, and an increase in the number of subsequent therapy options available. Indeed, similar increases in median OS can be seen in the control arm.

Given these challenges, how should we test effectiveness most appropriately in the first-line HER2-negative mBC setting? While Health Technology Assessment bodies worldwide accept PFS as a meaningful endpoint for clinical trials, progression-based outcomes are not recognized in Germany by the Institute for Quality and Efficiency in Health Services and the Joint Federal Committee (G-BA). These organizations focus on QoL, safety, OS, and morbidity, whereas PFS alone is not considered a meaningful endpoint, nor (in contrast with the clinical view) as an aspect of morbidity. The rationale for the G-BA's stance is that superior progressionbased outcomes evaluated by imaging are not considered to represent relevant benefits for patients. Patient relevance is accepted only if progression is recorded, for example, through symptoms perceptible to the patient. However, guidelines recommend assessing tumor burden every 8 weeks to allow prompt detection of metastatic progression, discontinuation of ineffective treatment with associated side effects, and prevention of tumor-associated symptoms that could be avoided by a change of treatment or strategy. ${ }^{31}$

Irrespective of surrogacy for OS, many believe that PFS is an important and relevant outcome for patients, associated with improved overall QoL, physical functioning, and emotional well-being. ${ }^{32}$ Extending PFS was ranked as more important than tumor shrinkage, limiting side effects, or treatment frequency in a questionnaire-based survey. Selfrated QoL was the highest after respondents had been told that their disease was responding to treatment. Therefore, progression-based parameters should generally be accepted as patient-relevant endpoints. Furthermore, changing therapy at progression affects patients' lives. A new therapy may be associated with new side effects and/or a new treatment schedule and mode of administration. The consequences 
Table I Overview of trials included in the analysis. Trials are ordered according to date enrollment began (earliest first)

\begin{tabular}{|c|c|c|c|c|}
\hline Trial & $\begin{array}{l}\text { Recruitment } \\
\text { period }\end{array}$ & Control arm (A) & Experimental arm (B) & $\begin{array}{l}\text { Missing information for } \\
\text { retrospective sample } \\
\text { size calculation }\end{array}$ \\
\hline HEPI $013^{10}$ & Sep 1990-Nov 1992 & $\begin{array}{l}\text { Cyclophosphamide, } \\
\text { methotrexate, } \\
\text { fluorouracil }\end{array}$ & $\begin{array}{l}\text { Cyclophosphamide, epirubicin, } \\
\text { fluorouracil }\end{array}$ & Yes $^{\mathrm{a}}$ \\
\hline SBG 9403"' & Feb 1995-Jan 1999 & Epirubicin & Vinorelbine + epirubicin & Yes \\
\hline Jassem et al $200 \mathrm{I}^{12}$ & Nov 1996-Apr 1998 & $\begin{array}{l}\text { Fluorouracil, doxorubicin, } \\
\text { cyclophosphamide }\end{array}$ & Doxorubicin + paclitaxel & Yes $^{\mathrm{a}}$ \\
\hline $\begin{array}{l}\text { von Minckwitz et al } \\
2005^{13}\end{array}$ & Nov 1996-Sep 200I & $\begin{array}{l}\text { Cyclophosphamide, } \\
\text { methotrexate, } \\
\text { fluorouracil }\end{array}$ & $\begin{array}{l}\text { Bendamustine, methotrexate, } \\
\text { fluorouracil }\end{array}$ & Yes \\
\hline $\begin{array}{l}\text { Bontenbal et al } \\
2005^{14}\end{array}$ & Mar 1997-Apr 2002 & $\begin{array}{l}\text { Fluorouracil, doxorubicin, } \\
\text { cyclophosphamide }\end{array}$ & Doxorubicin + docetaxel & No \\
\hline Albain et al $2008^{15}$ & Aug 1999-Apr 2002 & Paclitaxel & Gemcitabine + paclitaxel & No \\
\hline $\mathrm{E} 2100^{16-18}$ & Dec 200I-May 2004 & Paclitaxel & Bevacizumab + paclitaxel & No \\
\hline Sparano et al $2009^{19}$ & Sep 2004-Nov 2006 & Docetaxel & $\begin{array}{l}\text { Pegylated liposomal doxorubicin }+ \\
\text { docetaxel }\end{array}$ & No \\
\hline RIBBON-| $\left.\right|^{b, 20,2 \mid}$ & Dec 2005-Aug 2007 & Capecitabine & Bevacizumab + capecitabine & No \\
\hline AVADO ${ }^{c, 22}$ & Mar 2006-Apr 2007 & Docetaxel & Bevacizumab + docetaxel & No \\
\hline $\begin{array}{l}\text { ATX (BOOG } \\
2006-06)^{23}\end{array}$ & Jun 2007-Dec 2010 & $\begin{array}{l}\text { Paclitaxel + bevacizumab } \\
\text { induction, then } \\
\text { bevacizumab maintenance }\end{array}$ & $\begin{array}{l}\text { Paclitaxel }+ \text { bevacizumab }+ \\
\text { capecitabine induction, then } \\
\text { bevacizumab and capecitabine } \\
\text { maintenance }\end{array}$ & No \\
\hline IMELDA $^{24}$ & Jul 2009-Mar 20II & $\begin{array}{l}\text { Docetaxel + bevacizumab } \\
\text { induction, then } \\
\text { bevacizumab maintenance }\end{array}$ & $\begin{array}{l}\text { Docetaxel + bevacizumab induction, } \\
\text { then capecitabine + bevacizumab } \\
\text { maintenance }\end{array}$ & No \\
\hline MERiDiAN ${ }^{25,26}$ & Aug 2012-Dec 2013 & Paclitaxel & Paclitaxel + bevacizumab & No \\
\hline
\end{tabular}

Notes: ${ }^{a}$ Total study duration missing. 'Data reported for capecitabine cohort (anthracycline/taxane cohort not included because of heterogeneity of chemotherapy backbone). 'Data reported for comparison of bevacizumab $15 \mathrm{mg} / \mathrm{kg}$ vs placebo (bevacizumab $7.5 \mathrm{mg} / \mathrm{kg}$ not included in this analysis as there was no significant improvement in the primary endpoint) but events/patients for reported sample size calculations include all three treatment arms as reported in the statistical design section of the publication.

Abbreviations: NR, not reported; OS, overall survival; PFS, progression-free survival; TTP, time to disease progression.

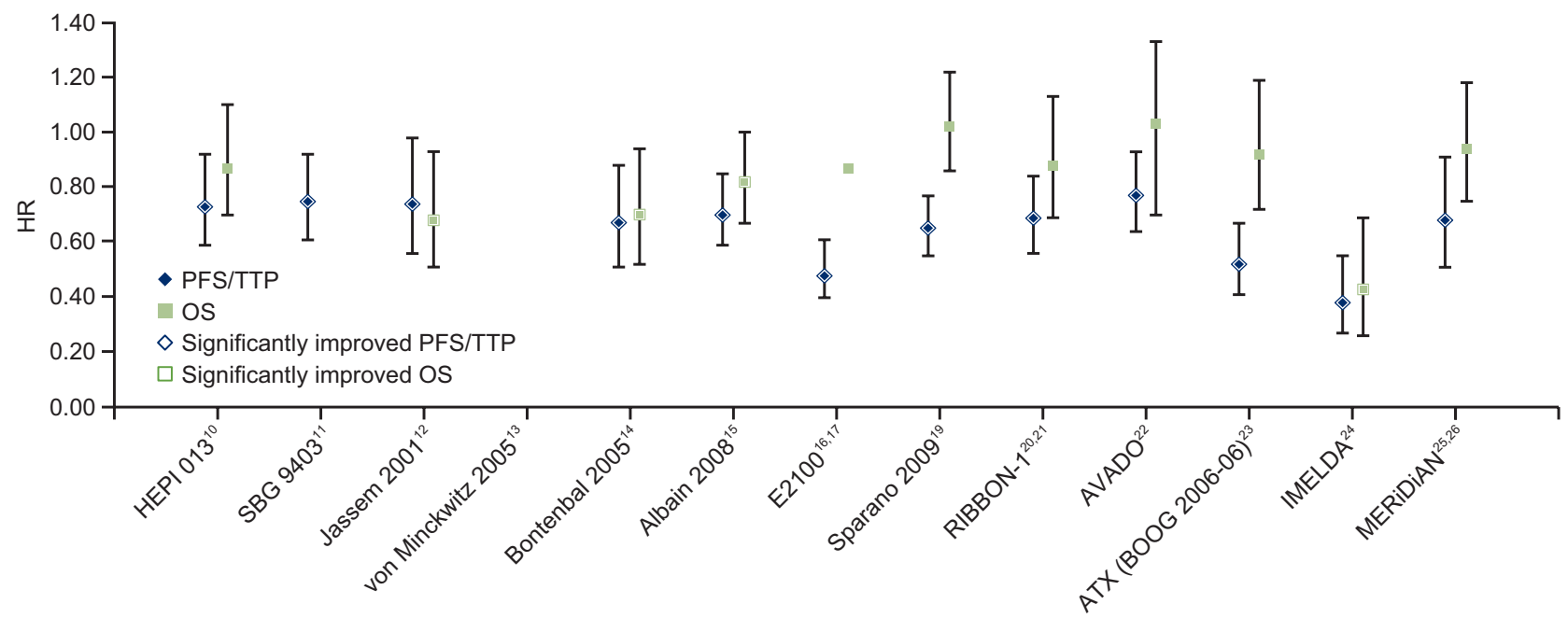

Figure I Summary of PFS/TTP and OS HRs across trials. Vertical bars represent $95 \% \mathrm{Cls}$, except for MERiDiAN, which shows the $99 \% \mathrm{Cl}$ reported for this coprimary endpoint.

Abbreviations: OS, overall survival; PFS, progression-free survival; TTP, time to progression. 


\begin{tabular}{|c|c|c|c|c|c|c|c|}
\hline \multicolumn{3}{|c|}{ Reported sample size calculation } & \multirow{2}{*}{$\begin{array}{l}\text { Primary } \\
\text { endpoint }\end{array}$} & \multirow{2}{*}{$\begin{array}{l}\text { Data cutoff } \\
\text { for primary } \\
\text { analysis }\end{array}$} & \multirow{2}{*}{$\begin{array}{l}\text { HR for PFS/ } \\
\text { TTP }(95 \% \mathrm{CI})\end{array}$} & \multirow{2}{*}{$\begin{array}{l}\text { HR for OS } \\
(95 \% \mathrm{Cl})\end{array}$} & \multirow{2}{*}{$\begin{array}{l}\text { Median duration of } \\
\text { follow-up, months }\end{array}$} \\
\hline $\begin{array}{l}\text { Assumed } \\
\text { HR }\end{array}$ & $\begin{array}{l}\text { Power, } \\
\%\end{array}$ & $\begin{array}{l}\text { Events/ } \\
\text { patients }\end{array}$ & & & & & \\
\hline 0.73 & 80 & $155 / 420$ & TTP & NR & $0.73(0.59-0.92)$ & $0.87(0.70-1.10)$ & $>20$ \\
\hline NR & NR & NR & PFS & NR & $0.75(0.61-0.92)$ & NR & $42(\mathrm{~A}) / 43(\mathrm{~B})$ \\
\hline 0.67 & 80 & $192 / 260$ & TTP & NR & $0.74(0.56-0.98)$ & $0.68(0.5 \mathrm{I}-0.93)$ & 29 \\
\hline NR & NR & $N R / 296$ & TTP & NR & NR & NR & NR \\
\hline 0.67 & 80 & $201 / 260$ & TTP & NR & $0.67(0.5 \mathrm{I}-0.88)$ & $0.70(0.52-0.94)$ & 27 (OS)/I4 (TTP) \\
\hline 0.75 & 80 & $377 / 526$ & OS & NR & $0.70(0.59-0.85)$ & $0.82(0.67-1.00)$ & NR \\
\hline 0.75 & 85 & $546 / 685$ & PFS & $\begin{array}{l}\text { Feb 9, } 2005 \\
\text { (PFS)/Oct } 21, \\
2006 \text { (OS) }\end{array}$ & $0.48(0.40-0.6 \mathrm{I})$ & 0.87 (NR) & NR \\
\hline 0.77 & $>80$ & $485 / 720$ & TTP & NR & $0.65(0.55-0.77)$ & $1.02(0.86-1.22)$ & NR \\
\hline 0.75 & 80 & $405 / 600$ & PFS & Jul 3I, 2008 & $0.69(0.56-0.84)$ & $0.88(0.69-1.13)$ & 16 (PFS)/23 (OS) \\
\hline 0.70 & 80 & $430 / 669$ & PFS & NR & $0.77(0.64-0.93)$ & I.03 (0.70-I.33) & 25 \\
\hline 0.81 & 80 & $N R / 303$ & PFS & Apr 26, 2013 & $0.52(0.4 I-0.67)$ & $0.92(0.72-1.19)$ & 41 \\
\hline 0.70 & 80 & $244 / 290$ & PFS & Oct 4,2013 & $0.38(0.27-0.55)$ & $0.43(0.26-0.69)$ & $30(\mathrm{~A}) / 32(\mathrm{~B})$ \\
\hline 0.67 & 85 & $326 / 480$ & PFS & $\begin{array}{l}\text { Nov } 30,2014 \\
\text { (PFS)/Apr 28, } \\
2017 \text { (OS) }\end{array}$ & $\begin{array}{l}0.68(99 \% \mathrm{Cl} \\
0.5 \mathrm{I}-0.9 \mathrm{I})\end{array}$ & $0.94(0.75-1.18)$ & $\begin{array}{l}\text { I5 (PFS)/24 (A) } \\
\text { and } 23(\mathrm{~B})(\mathrm{OS})\end{array}$ \\
\hline
\end{tabular}

Table 2 Summary of trial outcomes

\begin{tabular}{|c|c|c|c|c|c|c|c|c|}
\hline \multirow[t]{2}{*}{ Trial } & \multirow{2}{*}{$\begin{array}{l}\text { Total no } \\
\text { of patients } \\
\text { in trial }\end{array}$} & \multicolumn{3}{|c|}{$\begin{array}{l}\text { Observed median, months } \\
\text { (arm A vs arm B) }\end{array}$} & \multicolumn{2}{|c|}{$\begin{array}{l}\text { Retrospectively } \\
\text { calculated sample size }\end{array}$} & \multicolumn{2}{|c|}{ Factor $(x)$} \\
\hline & & PFS & TTP & OS & PFS/TTP & OS & $\begin{array}{l}\text { OS } \\
\text { sample } \\
\text { size/N }\end{array}$ & $\begin{array}{l}\text { OS/(PFS/TTP) } \\
\text { sample size }\end{array}$ \\
\hline HEPI $013^{10}$ & 460 & - & 6.3 vs 8.7 & 18.2 vs 20.1 & 360 & 5,906 & 12.8 & 16.4 \\
\hline SBG 9403'" & 387 & 8.2 vs 10.1 & - & 18.0 vs 19.1 & 788 & 11,988 & 31.0 & 15.2 \\
\hline Jassem et al $200 \mathrm{I}^{12}$ & 267 & - & 6.2 vs 8.3 & 18.3 vs 23.3 & 506 & 1,402 & 5.3 & 2.8 \\
\hline von Minckwitz et al $2005^{13}$ & 345 & - & 6.7 vs 8.2 & - & 792 & - & - & - \\
\hline Bontenbal et al $2005^{14}$ & 216 & - & 6.6 vs 8.0 & 16.2 vs 22.6 & I,022 & 476 & 2.2 & 0.5 \\
\hline Albain et al $2008^{15}$ & 529 & - & 4.0 vs 6.1 & 15.8 vs 18.6 & 168 & 1,404 & 2.7 & 8.4 \\
\hline $\mathrm{E} 2100^{16,17}$ & 722 & 5.8 vs 11.3 & - & 24.8 vs 26.5 & 74 & 10,396 & 14.4 & 140.5 \\
\hline Sparano et al $2009^{19}$ & 751 & - & 7.0 vs 9.8 & 20.6 vs 20.5 & 294 & $1,847,626$ & 2460.2 & 6284.4 \\
\hline RIBBON-| $\left.\right|^{20,21}$ & 615 & 5.7 vs 8.6 & - & 22.8 vs 25.7 & 264 & 6,000 & 9.8 & 22.7 \\
\hline AVADO ${ }^{\mathrm{a}, 22}$ & 488 & 8.2 vs 10.1 & - & 31.9 vs 30.2 & 806 & 21,136 & 43.3 & 26.2 \\
\hline ATX (BOOG 2006-06) $)^{23}$ & 312 & 8.4 vs 11.2 & - & 23.1 vs 24.2 & 440 & 24,178 & 77.5 & 55.0 \\
\hline IMELDA $^{24}$ & 185 & $4.3^{\mathrm{a}} \mathrm{vs} 11.9^{\mathrm{a}}$ & - & $23.7^{\mathrm{a}}$ vs $39.0^{\mathrm{a}}$ & 32 & 214 & 1.2 & 6.7 \\
\hline MERiDiAN ${ }^{25,26}$ & 481 & 8.8 vs 11.0 & - & 25.8 vs 28.8 & 866 & 6,728 & 14.0 & 7.8 \\
\hline
\end{tabular}

Notes: aedian values are not comparable with the other trials, first because PFS and OS were calculated from the time of randomization to maintenance therapy rather than the start of first-line therapy, and second because only patients with response or stable disease after induction therapy were included in the randomized population. Abbreviations: OS, overall survival; PFS, progression-free survival; TTP, time to disease progression. 


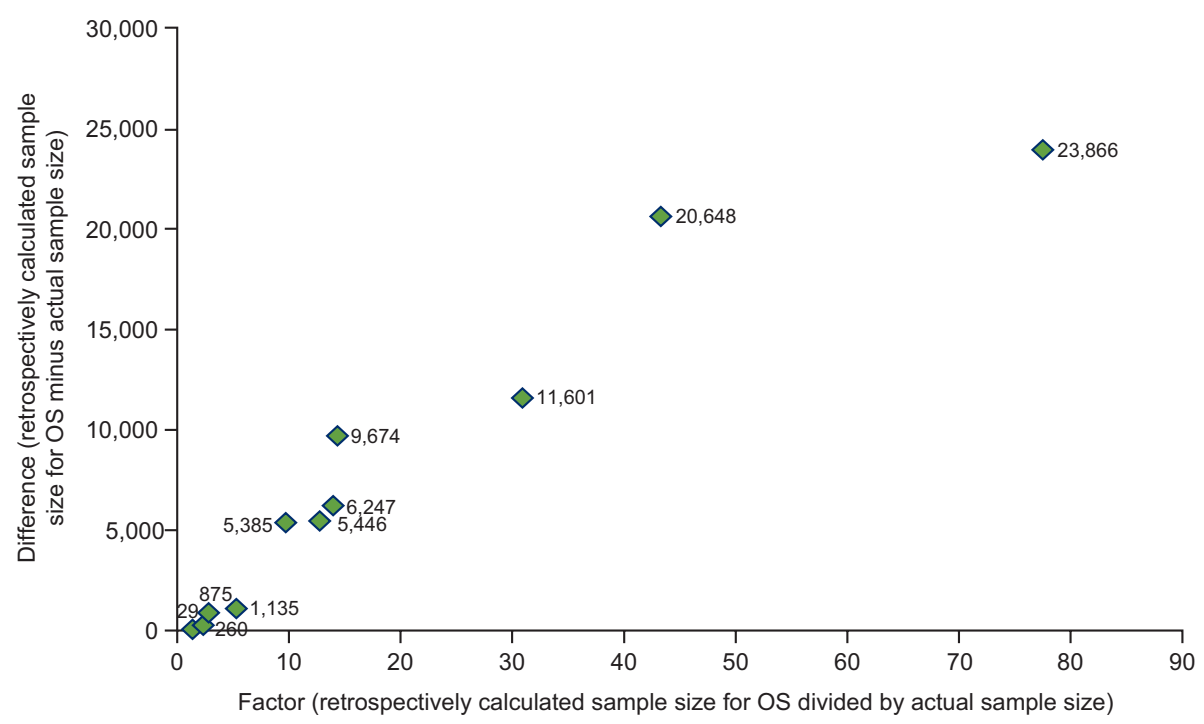

Figure 2 Additional patients required to show an OS benefit ${ }^{\mathrm{a}}$.

Note: ${ }^{a}$ One study ${ }^{19}$ is not shown on the figure as the numbers are so large $(x=2,460.2$, retrospectively calculated increase in sample size $=1,846,875)$.

Abbreviation: OS, overall survival.

of disease progression are depressive reactions, grief, and despair. The possibility of tumor control is the most important reason for patients agreeing to systemic therapy. ${ }^{33}$ Fear of disease progression is the most commonly reported psychological burden in patients. ${ }^{34}$

We acknowledge that PFS is not a perfect endpoint, potentially being influenced by assessment intervals, choice of target lesions, and measurement technology. Some of these challenges are overcome by Independent Central Review, which is important for accepting PFS as an endpoint. Regarding the limitations of OS, several elegant biostatistical methods have been developed to account for crossover, such as inverse probability of censoring weighting (IPCW) and the rank-preserving structural failure time (RPSFT) model. ${ }^{35,36}$ However, these approaches are not flawless: IPCW assumes that there are no unknown or unmeasured confounding factors that could influence crossover and OS, whereas RPSFT assumes that the effect of treatment is constant across time and/or treatment lines. No single validated standard for statistical correction of crossover has been established in settings with long postprogression survival.

With the increasing use of maintenance therapies, eg, in ovarian cancer, alternatives to PFS and OS have emerged, including intermediate endpoints such as time to second progression or time to first or second subsequent therapy. ${ }^{37}$ These endpoints merit consideration in future trial designs in HER2-negative mBC. For trials evaluating endocrine therapies, time to first chemotherapy can also be a valuable endpoint, with clear patient relevance. Alternative endpoints used in other tumor types include quality-adjusted time without symptoms or toxicity and quality-adjusted PFS. However, it is essential that any endpoint is clearly defined and that the precise definition is used consistently across trials measuring the effect of treatment. ${ }^{38}$ Changes in molecular markers may also be of interest as surrogate endpoints.

In an attempt to quantify the medical benefits of new drugs, composite scales including pharmacoeconomic parameters have been introduced, such as the European Society for Medical Oncology Magnitude of Clinical Benefit Scale (ESMO-MCBS) ${ }^{39}$ and the American Society of Clinical Oncology Value Framework. ${ }^{40} \mathrm{~A}$ recent survey indicated that many trials demonstrating statistically significant improvements in efficacy did not meet the ESMO-MCBS clinical benefit threshold, ${ }^{41}$ particularly trials in the palliative setting.

The challenge of large sample sizes required to show OS improvement in clinical trials has been accompanied by increased interest in real-world data (RWD). In some cases, RWD evaluation has suggested improved OS from a treatment despite the lack of OS benefit in prospective randomized clinical trials. ${ }^{42}$ The main advantages of RWD are the very large sample sizes available for analysis and inclusion of broader, more heterogeneous patient populations with common comorbidities than is possible in a clinical trial, reflecting populations presenting in routine oncology practice. However, there are many limitations and even with sophisticated statistical methodology, RWD are exposed to important potential biases. ${ }^{43}$ Therefore, RWD can be viewed 
only as complementary to randomized clinical trials, not as an alternative.

A limitation of our analysis is the focus on chemotherapy and antiangiogenic agents. Numerous ongoing trials in the first-line HER2-negative $\mathrm{mBC}$ setting are evaluating cancer immunotherapy agents, which have a different mode of action and thus may exhibit different effects on PFS and OS. Furthermore, many of these trials focus on triple-negative $\mathrm{mBC}$, a slightly more homogeneous population with shorter OS expectancy, shorter postprogression survival, and fewer treatment options after progression. All of these factors may affect the ability to demonstrate a significant OS effect, and, therefore, the patterns observed in our analysis may not predict future trials of cancer immunotherapy. Interestingly, several ongoing Phase III trials of immunotherapy in triplenegative $\mathrm{mBC}$ evaluate $\mathrm{OS}$ as the (co)primary endpoint. Another potential criticism is that the proportion of patients completing treatment is not taken into account. This information is missing in some of the publications, particularly in the older trials, but may have an impact on outcomes.

\section{Conclusion}

Although there are many reasons why OS is an attractive endpoint in trials of first-line therapy for HER2-negative $\mathrm{mBC}$, it has limitations. Designing trials to test potential new treatments for HER2-negative $\mathrm{mBC}$ is challenging and requires a balance of regulatory acceptability, feasibility, and realistic medical assumptions to calculate sample sizes, which can be particularly difficult in heterogeneous study populations with long postprogression survival and heterogeneous subsequent therapies. The magnitude of OS benefit likely to be considered as clinically (as well as statistically) significant depends on disease biology and risk. For example, in patients with triple-negative $\mathrm{mBC}$, a 3-month improvement in median OS is undoubtedly meaningful, whereas in hormone receptor-positive $\mathrm{mBC}$, a larger (6-month) improvement may be required to provide convincing meaningful benefit. In the current environment amid soaring costs and fierce competition, ${ }^{44}$ it is probably unrealistic to aim for trials demonstrating statistically significant OS improvement in this setting, except for trials in very specific poor prognosis populations. Ultimately, identification of robust alternative endpoints reflecting relevant patient benefits remains critical.

\section{Data availability}

All data used for the analyses reported in this paper are taken from the cited publications.

\section{Acknowledgments}

Medical writing support was provided by Jennifer Kelly, MA (Medi-Kelsey Ltd, Ashbourne, UK) and funded by Roche Pharma AG. This work was funded by Roche Pharma AG, Grenzach-Wyhlen, Germany. A poster reporting a preliminary analysis of this topic was presented at the European Society for Medical Oncology (ESMO) congress in Madrid, Spain; 8-12 September, 2017. The abstract from the poster was published in the congress proceedings. ${ }^{45}$

\section{Disclosure}

SKü and CJ have participated on advisory boards for Roche Pharma AG. VM has participated on advisory boards for Amgen, AstraZeneca, Celgene, Daiichi Sankyo, Eisai, Genomic Health, Nektar, Novartis, Pfizer, Pierre Fabre, Roche, and Teva. SK1 is an employee of Roche Pharma AG. MPL has participated on advisory boards for AstraZeneca, MSD, Novartis, Pfizer, Genomic Health, and Roche, and has received honoraria for lectures from Lilly, Roche, Novartis, Pfizer, Genomic Health, AstraZeneca, Medac, and Eisai. AS has declared no conflict of interest and the other authors report no other conflicts of interest in this work.

\section{References}

1. Mendes D, Alves C, Afonso N, et al. The benefit of HER2-targeted therapies on overall survival of patients with metastatic HER2positive breast cancer - a systematic review. Breast Cancer Res. 2015; 17:140.

2. Di Leo A, Bleiberg H, Buyse M. Overall survival is not a realistic end point for clinical trials of new drugs in advanced solid tumors: a critical assessment based on recently reported phase III trials in colorectal and breast cancer. J Clin Oncol. 2003;21(10):2045-2047.

3. Sargent DJ, Hayes DF. Assessing the measure of a new drug: is survival the only thing that matters? J Clin Oncol. 2008;26(12):1922-1923.

4. Broglio KR, Berry DA. Detecting an overall survival benefit that is derived from progression-free survival. J Natl Cancer Inst. 2009;101(23):1642-1649.

5. Amir E, Seruga B, Kwong R, Tannock IF, Ocaña A. Poor correlation between progression-free and overall survival in modern clinical trials: are composite endpoints the answer? Eur J Cancer. 2012;48(3):385-388.

6. Korn EL, Freidlin B, Abrams JS. Overall survival as the outcome for randomized clinical trials with effective subsequent therapies. J Clin Oncol. 2011;29(17):2439-2442.

7. Adunlin G, Cyrus JW, Dranitsaris G. Correlation between progressionfree survival and overall survival in metastatic breast cancer patients receiving anthracyclines, taxanes, or targeted therapies: a trial-level meta-analysis. Breast Cancer Res Treat. 2015;154(3):591-608.

8. Liu L, Chen F, Zhao J, Yu H. Correlation between overall survival and other endpoints in metastatic breast cancer with second- or third-line chemotherapy: literature-based analysis of 24 randomized trials. Bull Cancer. 2016;103(4):336-344.

9. Forsythe A, Chandiwana D, Barth J, Thabane M, Baeck J, Tremblay G. Progression-free survival/time to progression as a potential surrogate for overall survival in HR+, HER2- metastatic breast cancer. Breast Cancer. 2018;10:69-78. 
10. Ackland SP, Anton A, Breitbach GP, et al. Dose-intensive epirubicin-based chemotherapy is superior to an intensive intravenous cyclophosphamide, methotrexate, and fluorouracil regimen in metastatic breast cancer: a randomized multinational study. J Clin Oncol. 2001;19(4):943-953.

11. Ejlertsen B, Mouridsen HT, Langkjer ST, Andersen J, Sjöström J, Kjaer M. Phase III study of intravenous vinorelbine in combination with epirubicin versus epirubicin alone in patients with advanced breast cancer: a Scandinavian Breast Group Trial (SBG9403). J Clin Oncol. 2004;22(12):2313-2320.

12. Jassem J, Pieńkowski T, Płuzańska A, et al. Doxorubicin and paclitaxel versus fluorouracil, doxorubicin, and cyclophosphamide as first-line therapy for women with metastatic breast cancer: final results of a randomized phase III multicenter trial. J Clin Oncol. 2001;19:1707-1715.

13. von Minckwitz G, Chernozemsky I, Sirakova L, et al. Bendamustine prolongs progression-free survival in metastatic breast cancer (MBC): a phase III prospective, randomized, multicenter trial of bendamustine hydrochloride, methotrexate and 5-fluorouracil (BMF) versus cyclophosphamide, methotrexate and 5-fluorouracil (CMF) as first-line treatment of MBC. Anticancer Drugs. 2005;16(8):871-877.

14. Bontenbal M, Creemers GJ, Braun HJ, et al. Phase II to III study comparing doxorubicin and docetaxel with fluorouracil, doxorubicin, and cyclophosphamide as first-line chemotherapy in patients with metastatic breast cancer: results of a Dutch Community Setting Trial for the Clinical Trial Group of the Comprehensive Cancer Centre. J Clin Oncol. 2005;23(28):7081-7088.

15. Albain KS, Nag SM, Calderillo-Ruiz G, et al. Gemcitabine plus paclitaxel versus paclitaxel monotherapy in patients with metastatic breast cancer and prior anthracycline treatment. J Clin Oncol. 2008;26(24):3950-3957.

16. Gray R, Bhattacharya S, Bowden C, Miller K, Comis RL. Independent review of E2100: a phase III trial of bevacizumab plus paclitaxel versus paclitaxel in women with metastatic breast cancer. J Clin Oncol. 2009;27(30):4966-4972.

17. Cameron D. Bevacizumab in the first-line treatment of metastatic breast cancer. Eur J Cancer. 2008;6(6):21-28.

18. Miller K, Wang M, Gralow J, et al. Paclitaxel plus bevacizumab versus paclitaxel alone for metastatic breast cancer. $N$ Engl J Med. 2007;357(26):2666-2676.

19. Sparano JA, Makhson AN, Semiglazov VF, et al. Pegylated liposomal doxorubicin plus docetaxel significantly improves time to progression without additive cardiotoxicity compared with docetaxel monotherapy in patients with advanced breast cancer previously treated with neoadjuvant-adjuvant anthracycline therapy: results from a randomized phase III study. J Clin Oncol. 2009;27(27):4522-4529.

20. Robert NJ, Diéras V, Glaspy J, et al. RIBBON-1: randomized, doubleblind, placebo-controlled, phase III trial of chemotherapy with or without bevacizumab for first-line treatment of human epidermal growth factor receptor 2-negative, locally recurrent or metastatic breast cancer. J Clin Oncol. 2011;29(10):1252-1260.

21. Roche data on file. AVF3694g clinical study report addendum. 2009.

22. Miles DW, Chan A, Dirix LY, et al. Phase III study of bevacizumab plus docetaxel compared with placebo plus docetaxel for the first-line treatment of human epidermal growth factor receptor 2-negative metastatic breast cancer. J Clin Oncol. 2010;28(20):3239-3247.

23. Lam SW, de Groot SM, Honkoop AH, et al. Paclitaxel and bevacizumab with or without capecitabine as first-line treatment for HER2-negative locally recurrent or metastatic breast cancer: a multicentre, open-label, randomised phase 2 trial. Eur J Cancer. 2014;50(18):3077-3088.

24. Gligorov J, Doval D, Bines J, et al. Maintenance capecitabine and bevacizumab versus bevacizumab alone after initial first-line bevacizumab and docetaxel for patients with HER2-negative metastatic breast cancer (IMELDA): a randomised, open-label, phase 3 trial. Lancet Oncol. 2014;15(12):1351-1360.

25. Miles D, Cameron D, Bondarenko I, et al. Bevacizumab plus paclitaxel versus placebo plus paclitaxel as first-line therapy for HER2-negative metastatic breast cancer (MERiDiAN): a double-blind placebo-controlled randomised phase III trial with prospective biomarker evaluation. Eur J Cancer. 2017;70:146-155.
26. Miles D, Cameron D, Hilton M, Garcia J, O’Shaughnessy J. Overall survival in MERiDiAN, a double-blind placebo-controlled randomised phase III trial evaluating first-line bevacizumab plus paclitaxel for HER2negative metastatic breast cancer. Eur J Cancer. 2018;90:153-155.

27. Tan A, Porcher R, Crequit P, Ravaud P, Dechartres A. Differences in treatment effect size between overall survival and progression-free survival in immunotherapy trials: a meta-epidemiologic study of trials with results posted at ClinicalTrials.gov. J Clin Oncol. 2017;35(15): 1686-1694.

28. Saad ED, Katz A. Progression-free survival and time to progression as primary end points in advanced breast cancer: often used, sometimes loosely defined. Ann Oncol. 2009;20(3):460-464.

29. Burzykowski T, Buyse M, Piccart-Gebhart MJ, et al. Evaluation of tumor response, disease control, progression-free survival, and time to progression as potential surrogate end points in metastatic breast cancer. J Clin Oncol. 2008;26(12):1987-1992.

30. Forsythe A, Chandiwana D, Barth J, et al. Is progression-free survival a more relevant endpoint than overall survival in first-line HR+/HER2metastatic breast cancer? Cancer Manag Res. 2018;10:1015-1025.

31. Thill M, Liedtke C, Solomayer EF, Müller V, Janni W, Schmidt M. AGO recommendations for the diagnosis and treatment of patients with advanced and metastatic breast cancer: update 2017. Breast Care. 2017;12(3):184-191.

32. Hurvitz SA, Lalla D, Crosby RD, Mathias SD. Use of the metastatic breast cancer progression (MBC-P) questionnaire to assess the value of progression-free survival for women with metastatic breast cancer. Breast Cancer Res Treat. 2013;142(3):603-609.

33. Grunfeld EA, Maher EJ, Browne S, et al. Advanced breast cancer patients' perceptions of decision making for palliative chemotherapy. J Clin Oncol. 2006;24(7):1090-1098.

34. Herschbach P, Keller M, Knight L, et al. Psychological problems of cancer patients: a cancer distress screening with a cancer-specific questionnaire. Br J Cancer. 2004;91(3):504-511.

35. Ishak KJ, Proskorovsky I, Korytowsky B, Sandin R, Faivre S, Valle J. Methods for adjusting for bias due to crossover in oncology trials. Pharmacoeconomics. 2014;32(6):533-546.

36. Korhonen P, Zuber E, Branson M, et al. Correcting overall survival for the impact of crossover via a rank-preserving structural failure time (RPSFT) model in the RECORD-1 trial of everolimus in metastatic renal-cell carcinoma. J Biopharm Stat. 2012;22(6):1258-1271.

37. Karam A, Ledermann JA, Kim JW, et al. Fifth Ovarian Cancer Consensus Conference of the Gynecologic Cancer InterGroup: first-line interventions. Ann Oncol. 2017;28:711-717.

38. Gourgou-Bourgade S, Cameron D, Poortmans P, et al. Guidelines for time-to-event end point definitions in breast cancer trials: results of the DATECAN initiative (Definition for the Assessment of Time-to-event Endpoints in CANcer trials). Ann Oncol. 2015;26(12):2505-2506.

39. Cherny NI, Dafni U, Bogaerts J, et al. ESMO-Magnitude of Clinical Benefit Scale version 1.1. Ann Oncol. 2017;28(10):2340-2366.

40. Schnipper LE, Davidson NE, Wollins DS, et al. American Society of Clinical Oncology Statement: a conceptual framework to assess the value of cancer treatment options. J Clin Oncol. 2015;33(23):2563-2577.

41. Del Paggio JC, Azariah B, Sullivan R, et al. Do contemporary randomized controlled trials meet ESMO thresholds for meaningful clinical benefit? Ann Oncol. 2017;28(1):157-162.

42. Delaloge S, Pérol D, Courtinard C, et al. Paclitaxel plus bevacizumab or paclitaxel as first-line treatment for HER2-negative metastatic breast cancer in a multicenter national observational study. Ann Oncol. 2016;27(9):1725-1732.

43. Buyse M, Vansteelandt S. The potential and perils of observational studies. Ann Oncol. 2017;28(1):182.

44. Piccart M, Pondé N. Cancer drugs, survival and ethics: a critical look from the inside. ESMO Open. 2017;1(6):e000149.

45. Kümmel S, Jackisch C, Müller V, Schneeweiss A, Klawitter S, Lux MP. Can contemporary trials in HER2-negative metastatic breast cancer (mBC) detect overall survival (OS) benefit? Ann Oncol. 2017;28(Suppl 5): v86-v87. 


\section{Supplementary material}

Table SI Search strategy for identification of eligible trials

\begin{tabular}{|c|c|c|}
\hline Set\# & Searched for & Results \\
\hline $\mathrm{SI}$ & MESH.EXACT.EXPLODE(“Breast Neoplasms”) & 260,430 \\
\hline S2 & $\begin{array}{l}\text { ti,ab((breast OR mamma*) NEAR/2 (cancer* OR carcinoma* OR tumo* OR neoplasm* or neoplasm* or malignanc*)) } \\
\text { AND dstat.exact("Publisher" OR “In Process" OR “PubMed not MEDLINE” OR “In Data Review”) }\end{array}$ & 32,411 \\
\hline S3 & sl or s2 & 292,808 \\
\hline S4 & ti,ab(metasta* or mBC or dissemin* or spread or advanced) AND s3 & 66,764 \\
\hline S5 & MESH.EXACT.EXPLODE(“Neoplasm Metastasis”) AND s3 & 31,899 \\
\hline S6 & $\mathrm{s} 4$ or $\mathrm{s} 5$ & 78,519 \\
\hline S7 & s6 AND rtype.exact(“Randomized Controlled Trial”) & 2,832 \\
\hline S8 & all(randomized) AND s6 & 5,803 \\
\hline S9 & all(placebo) AND s6 & 475 \\
\hline SIO & s7 or $s 8$ or $s 9$ & 5,920 \\
\hline SII & (s7 or s8 or s9) AND rtype.exact(“Clinical Trial, Phase III” OR “Clinical Trial, Phase II”) & 969 \\
\hline $\mathrm{SI2}$ & (ti,ab(phase $\mathrm{p} / 2 \mathrm{III}[* \mathrm{I}]$ or phase $\mathrm{p} / 23\left[^{*} \mathrm{I}\right]$ or phase $\mathrm{p} / 2 \mathrm{II}[* 4]$ or phase $\left.\mathrm{p} / 22\left[{ }^{*} 4\right]\right)$ AND s I0) & $\mathrm{I}, 468$ \\
\hline $\mathrm{SI3}$ & (s।2) and (dstat.exact(“Publisher" OR “In Process” OR “PubMed not MEDLINE” OR “In Data Review”)) & 126 \\
\hline $\mathrm{SI} 4$ & $(s||$ or $s \mid 3)$ & 1,095 \\
\hline SI5 & $((\mathrm{s} \mid \mathrm{I}$ or $\mathrm{s} \mid 3))$ and $(\mathrm{pd}(20|4-20| 8))$ & 348 \\
\hline
\end{tabular}

Cancer Management and Research

\section{Publish your work in this journal}

Cancer Management and Research is an international, peer-reviewed open access journal focusing on cancer research and the optimal use of preventative and integrated treatment interventions to achieve improved outcomes, enhanced survival and quality of life for the cancer patient. The manuscript management system is completely online and includes 